\title{
TRATAMIENTO DE NEVUS DE OTA CON LÁSER Q-SWITCHED ALEXANDRITA DE 755 NM
}

\author{
TREATMENT OF OV NEVUS WITH Q-SWITCHED ALEXANDRITA LASER 755 NM
}

Julio Cesar Negron-Beuzeville ${ }^{1, b}$, Edmundo Saco M.2,b, Iván Hernández P.,4,a

\begin{abstract}
RESUMEN
Introducción: El nevus de Ota es un hamartoma melanocítico dérmico benigno de aspecto macular, mal delimitado y en parche de color negrogrisáceo que generalmente afecta unilateralmente la primera y segunda rama del trigémino. Objetivo: Evaluar la eficacia, seguridad y los eventos adversos ocasionados por la aplicación de láser Alexandrita de $755 \mathrm{~nm}$ para el tratamiento del nevus de Ota. Métodos: Se trata de una serie de casos realizado entre enero del 2017 y diciembre 2018 con una muestra de 10 pacientes con una media de 29 años (de 16 a 40 años) y con diagnóstico clínico de nevus de Ota (NO) unilateral. Todos ellos con fototipo de piel tipo IV, tratados en la clínica de la piel. Se utilizó el láser Alex Trivantage Candela Q-switched Alexandrita de $755 \mathrm{~nm}$ con parámetros definidos de energía de de $6 \mathrm{j} / \mathrm{cm} 2$, frecuencia de $3 \mathrm{hz}$ y spot de $3 \mathrm{~mm}$. Los pacientes incluidos en este trabajo firmaron el consentimiento informado que incluye el uso de su registro fotográfico para fines científicos. Así mismo, el estudio fue autorizado por el comité de ética institucional. Se incluyeron a pacientes mayores de edad con diagnóstico de NO sin antecedentes de tratamiento para el mismo. Se excluyeron pacientes en tratamiento con retinoides orales, infecciones activas o lesiones sospechosas de malignidad, pacientes embarazadas o en periodo de lactancia y pacientes que no aceptaron el consentimiento informado. Resultados: La media de edad fue de 29 años., Los pacientes que completaron el tratamiento no presentaron repigmentación en la lesión tratada y el índice de satisfacción fue aceptable. Se observó que el promedio de sesiones para obtener resultados favorables varió entre 8 y 10 aplicaciones. Dentro de los efectos adversos más importantes resalta la hiperpigmentación postinflamatoria y la eliminación del estrato superficial de la piel post láser. Todos fueron controlados sin dejar lesiones residuales permanentes. Conclusión: El láser Q-switched Alexandrita de $755 \mathrm{~nm}$ es una tecnología efectiva para lograr un alto grado de despigmentación en el tratamiento del nevus de Ota y puede ser considerado como un tratamiento de primera línea para el manejo de esta patología.
\end{abstract}

Palabras clave: Alexandrita láser 755 nm; Nevus de Ota; Q-switched; Lesiones pigmentarias congénitas; Nevus congénito facial (fuente: DeCS BIREME).

\begin{abstract}
Introduction: Ota nevus is a benign dermal melanocytic hamartoma with a macular appearance, poorly defined and in a gray-black patch that generally affects the first and second branches of the trigeminal unilaterally. Objective: To evaluate the efficacy, safety and adverse events caused by the application of $755 \mathrm{~nm}$ Alexandrite laser for the treatment of Ota nevus. Methods: This is a series of cases with carried out between January 2017 and December 2018 on a sample of 10 patients with an average of 29 years (from 16 to 40 years old) and with a clinical diagnosis of unilateral (ON). All of them with type IV skin photo type, treated in the skin clinic. The Alex Trivantage Candela Q-switched Alexandrite $755 \mathrm{~nm}$ laser was used with defined energy parameters of $6 \mathrm{j} / \mathrm{cm} 2 \mathrm{and} 3 \mathrm{~Hz}$ frequency and $3 \mathrm{~mm}$ spot. All included patients signed the informed consent that includes the use of their photographic record for scientific purposes. Likewise, the study was authorized by the institutional ethics committee. Patients treated with oral retinoids, active infections or suspicious lesions of malignancy, pregnant or lactating patients and patients who did not accept informed consent were excluded. Results: The mean age was 29 years. The patients who completed the treatment did not show repigmentation in the treated lesion and the satisfaction index was acceptable. It was observed that the average of sessions to obtain favorable results varied between 8 and 10 applications. Among the most important adverse effects, post-inflammatory hyperpigmentation and the removal of the superficial layer of the post laser skin stand out. All were controlled without leaving permanent residual lesions. Conclusion: The $755 \mathrm{~nm}$ Alexandrite Q-switched laser is an effective technology to achieve a high degree of depigmentation in the treatment of Ota nevus and can be considered as a first-line treatment for the management of this pathology.
\end{abstract}

Key words: Alexandrite laser 755 nm; Ota nevus, Q-switched; Congenital pigmentary lesions; Congenital facial nevus (source: MeSH NLM).

\footnotetext{
${ }^{1}$ Clínica Lederma, Lima-Perú.

${ }^{2}$ Clínica D’Laser, Lima-Perú.

${ }^{3}$ Instituto Peruano de Ingeniería Celular y Manufactura Tisular - INGECEL, Lima-Perú.

4 Instituto de Investigación en Ciencias Biomédicas, Facultad de Medicina - Universidad Ricardo Palma, Lima-Perú.

a Cirujano plástico.

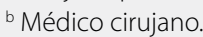

Citar como: Julio Cesar Negron-Beuzeville, Edmundo Saco M., Iván Hernández P. Tratamiento de Nevus de Ota con láser Q-Switched Alexandrita de 755 nm. Rev. Fac. Med. Hum. Enero 2020; 20(1):88-93. DOI 10.25176/RFMH.v20i1.2551 


\section{INTRODUCCIÓN}

El nevus de Ota (NO) es llamado así debido al que el dermatólogo japonés Ota, fue el primero en describir la entidad en 1936. ${ }^{(1)}$ Se trata de un hamartoma melanocítico dérmico benigno que se presenta a nivel facial, con predominio periorbitario, frontal, temporal, nariz y área ocular, pudiendo comprometer esclera, conjuntiva, cornea, e inclusive retina. ${ }^{(2)}$ Es una formación de aspecto macular mal delimitado en parche de color negrogrisáceo, que afecta la primera y segunda rama del trigémino. ${ }^{(3)}$ Aproximadamente el $90 \%$ de los casos del NO son unilaterales.

Se manifiesta desde la infancia como una hiperpigmentación que aumenta progresivamente en tamaño e intensidad de color hasta la pubertad(4) Aunque pueden estar presentes en un $1 \%$ de los neonatos, suelen iniciar su aparición a partir de los 6-12 meses e ir aumentando en número y tamaño hasta alrededor de los 25 años. ${ }^{(5)}$

EI NO puede presentarse en todas las razas, pero es más común en asiáticos, ${ }^{(6)}$ afecta al $0.5 \%$ de la población mundial y su presentación es mayor en mujeres con una frecuencia de 1 a $5 .{ }^{(7)}$

La etiopatogenia de esta melanocitosis dérmica es desconocida. Se han propuesto diferentes teorías, pero ninguna es totalmente satisfactoria. Hori Y. y Cols. ${ }^{(8)}$ ya desde el año 1984, proponían que su origen podría ser atribuido a dos posibles mecanismos: al descenso o migración de los melanocitos desde la epidermis o del bulbo piloso hacia la dermis o la reactivación de melanocitos dérmicos preexistentes, desencadenado por mecanismos inflamatorios o degenerativos. También, se ha propuesto que podría existir una influencia hormonal, ya que, por ejemplo, la aparición del NO es más típica en la pubertad. ${ }^{(9)}$ Los melanocitos están regidos por hormonas producidas a nivel de la hipófisis anterior, glándulas suprarrenales y ovarios además de los mecanismos en común de sus precursores. El diagnostico se realiza en consultorio, siendo pocas veces necesario la biopsia para la confirmación diagnóstica ${ }^{(10)}$ (Tabla 1).

Tabla 1. Clasificación del NO en función de la extensión de las lesiones.

\begin{tabular}{|l|l|}
\hline Clasificación & Características de presentación \\
\hline Tipo I & Pequeño: Órbitopalpebral y/o zigomático. \\
\hline Tipo II & Intermedio: Afecta las regiones orbitaria, zigomática y nasal. \\
\hline Tipo III & Bilateral. \\
\hline Tipo IV & \\
\hline
\end{tabular}

Adaptado de: Dermatología. Atlas, diagnóstico y tratamiento. Mc Graw Hill. $6 a$ Ed.

Los tratamientos actuales buscan mejorar la apariencia de los pacientes. El camuflaje es un tratamiento efectivo para mejorar la coloración y el tamaño. La crioterapia busca suprimir la función de los melanocitos a nivel epidérmico y destruir los melanocitos dérmicos. La dermoabrasión remueve la melanina de la epidermis y de la dermis superficial. Un método que actualmente ya no es indicado es la microcirugía, porque crea una cicatriz superficial. ${ }^{11}$

El láser ha revolucionado el tratamiento del NO. Para tratar estas lesiones, se han desarrollado láseres específicos de pigmento, entrelos cuales seencuentran: el láser Ruby Q-switched (694 nm), el Alexandrita Q-switched (755 nm), el Neodymium:YttriumAluminum-Garnet (1064 nm y $532 \mathrm{~nm}$ ), láser colorante pulsado $(510 \mathrm{~nm})$, láser vapor de cobre $(578 \mathrm{~nm})$. El láser Alexandrita Q-switched (755 nm) se basa en la fototermólisis selectiva que provee energía alta con pulsos de corta duración, que permiten crear una destrucción selectiva de los melanocitos.

Golberg y cols., ${ }^{12}$ fueron los primeros en reportar el uso de Q-switched Ruby para el tratamiento de NO. La eficacia de este tratamiento fue luego respaldada en un estudio que involucró 114 pacientes tratados con este láser. ${ }^{13}$ El efecto colateral fue la hiperpigmentación. Debido a que el NO mayormente se presenta en individuos con piel pigmentada, se debe tener cuidado al seleccionar la modalidad del láser y la fluencia para minimizar la lesión de los melanocitos no lesiónales. ${ }^{14}$

A comparación del láser Q-switched Ruby; el láser Q-switched Alexandrita tiene longitudes de onda más largas y es menos absorbido por la melanina, lo que evita el daño a la epidermis y permite una penetración más profunda; ${ }^{15}$ en consecuencia, puede 
selectivamente remover la pigmentación epidérmica y dérmica, por lo cual es ideal para el tratamiento de lesiones profundas como ésta, motivo de estudio.

Debido a ello, el objetivo de este estudio fue evaluar la eficacia, la seguridad y los efectos adversos en el tratamiento de NO con el láser Q-switched 755 nm Alex Trivantage - Candela.

\section{MÉTODOS}

Se trata de una serie de casos realizado entre enero del 2017 y diciembre 2018 con una muestra de 10 pacientes con una media de 29 años (de 16 a 40 años) y con diagnóstico clínico de nevus de Ota (NO) unilateral. Todos ellos con fototipo de piel tipo IV, tratados en la clínica de la piel. Se utilizó el láser Alex Trivantage Candela Q-switched Alexandrita de 755 $\mathrm{nm}$ con parámetros definidos de energía de de $6 \mathrm{j} /$ $\mathrm{cm} 2$, frecuencia de $3 \mathrm{hz}$ y spot de $3 \mathrm{~mm}$. Los pacientes incluidos en este trabajo firmaron el consentimiento informado que incluye el uso de su registro fotográfico para fines científicos. Se incluyeron a pacientes mayores de edad con diagnóstico de NO sin antecedentes de tratamiento para el mismo. Se excluyeron pacientes en tratamiento con retinoides orales, infecciones activas o lesiones sospechosas de malignidad, pacientes embarazadas o en periodo de lactancia y pacientes que no aceptaron el consentimiento informado.

Los registros fotográficos se dieron con una cámara Canon T3i bajo los mismos parámetros técnicos fotográficos al iniciar el tratamiento. A todos ellos se les aplico anestesia tópica en el área a tratar y se ocluye con plástico film por $30 \mathrm{~min}$. Luego se procedió a la aplicación del láser en el área de lesión. Posteriormente se colocaron packs fríos por 5 minutos y luego mupirocina en crema con betametasona de manera tópica. Como prescripción médica se indicó cremas con gentamicina / clotrimazol / betametasona cada 8 horas por 3 días, bepanthen (crema) cada 8 horas por 15 días, el 4to día se inició Isdin Ultra 90 (fotoprotector) de aplicación condicional a exposición y con horas referenciales 9am, 12 pm y 3pm por 30 días.

En todos los pacientes se evaluaron variables como; tamaño de la lesión, frecuencia y número de sesiones, si completó o no el tratamiento, así como efectos secundarios. Para evaluar el resultado del tratamiento se creó una escala en la cual se valoró la apreciación del paciente y la valoración del médico. Ésta escala solo muestra finalmente la valoración del cambio de acuerdo con la pigmentación, con lo cual se estimó si el tratamiento fue efectivo o no. Para ello se desarrolló una tabla cualitativa sencilla para el paciente y el operador, la cual contenía las variables de estudio y sus características incluyendo la reducción del tamaño de la lesión; la mejora en la pigmentación; la presencia de efectos colaterales (hiper o hipopigmentación) y la valoración de la lesión (depigmentación ó no)

En caso de presentarse hiperpigmentación post aplicación, se les recomendó la aplicación tópica de una fórmula magistral de Ácido Kojico 2\%, Ácido Mandélico $5 \%$, triamcinolona $0,025 \%$ de uso nocturno. De presentarse irritación a esta fórmula, se le indicó aplicación de paños con manzanilla fría de 3 a 5 minutos, cada 6 horas, durante 5 días. En casos de irritación intensa, se procedió a aplicar cada 12 horas una formulación con betametasona 0,05\% durante 3 días. Una vez superado este episodio, se reinició el uso de la formulación despigmentante de forma inter diaria, hasta la siguiente sesión de láser de acuerdo a protocolo.

\section{RESULTADOS}

Todos los pacientes atendidos fueron de sexo femenino, con fototipo IV, siendo el grupo etareo de 20 a 30 años el más representativo con 6 pacientes. Las áreas afectadas en todos los pacientes tratados se localizaron unilateralmente en pómulo y mejilla. Tres de los pacientes presentaban compromiso conjuntival y nasal (Figura 1). La mayoría de pacientes presentaron compromiso de dos o más localizaciones.

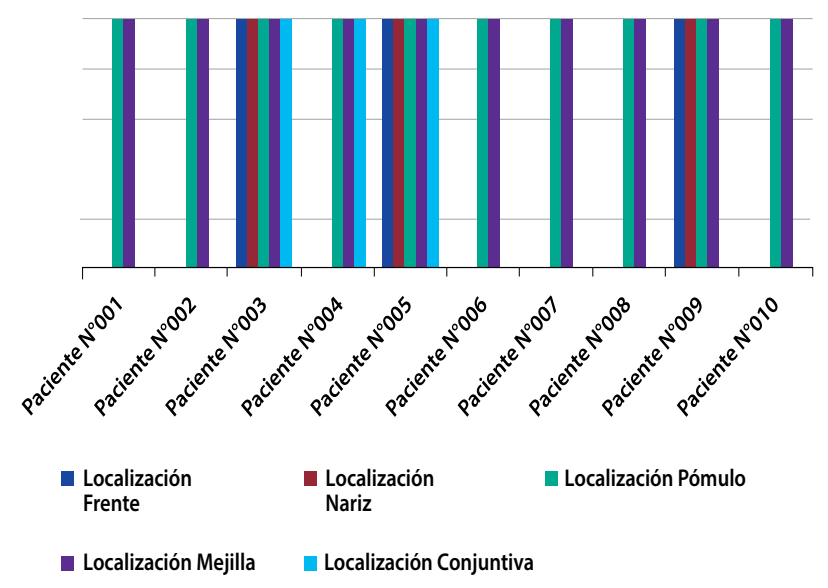

Figura 1. Detalle de áreas afectadas en relación a los casos presentados.

5 pacientes presentaban una lesión grande, mayor a $10 \mathrm{~cm} ; 4$ pacientes lesión de tamaño mediano $<$ de $10 \mathrm{~cm}$ y $>$ de $5 \mathrm{~cm}$; solo 1 paciente presentó una lesión menor a $5 \mathrm{~cm}$.

Por diferentes motivos, se generaron diversas frecuencias de sesiones y solo 4 pacientes respetaron lo acordado en cuanto al tiempo indicado entre sesiones de cada 30 días; 3 pacientes tuvieron frecuencia entre sesiones de 60 días; el resto, uno asistió cada 45 días, otro cada 90 días y el último cada 120 días. 
6 pacientes concluyeron de manera satisfactoria el tratamiento y 4 pacientes lo abandonaron a la $7 \mathrm{ma}$ sesión por diversos motivos, siendo el principal, la aparición de efectos colaterales y que, según la escala de valoración, no evidenciaban mejoría en su lesión.

Los pacientes que completaron el tratamiento fueron los que tuvieron de 8 a más sesiones y se evidenció que el tratamiento resultó satisfactorio a partir de la 8 va sesión. El $70 \%$ de pacientes presentó efectos colaterales, sin embargo, solo el $20 \%$ de este grupo no culminó el tratamiento; estos pacientes presentaron frecuencias de aplicación de láser cada 30 días y cada 120 días. (Tabla 2 )

Tabla 2. Frecuencia de sesiones, número de sesiones, abandono y efectos colaterales.

\begin{tabular}{|cccccc|}
\hline Caso & Frecuencia sesiones & Número de sesiones & Completo tratamiento & Efectos colaterales \\
\hline 1 & 60 días & 10 & $\mathrm{SI}$ & $\mathrm{SI}$ \\
\hline 2 & 30 días & 10 & $\mathrm{SI}$ & $\mathrm{SI}$ \\
\hline 3 & 60 días & 8 & $\mathrm{SI}$ & $\mathrm{SI}$ \\
\hline 4 & 120 días & 5 & $\mathrm{NO}$ & $\mathrm{SI}$ \\
\hline 5 & 60 días & 9 & $\mathrm{SI}$ & $\mathrm{NO}$ \\
\hline 8 & 45 días & 8 & $\mathrm{SI}$ & $\mathrm{SI}$ \\
\hline 9 & 30 días & 6 & $\mathrm{NO}$ & $\mathrm{NO}$ \\
\hline 10 & 90 días & 30 días & 5 & $\mathrm{NO}$ & $\mathrm{SI}$ \\
\hline
\end{tabular}

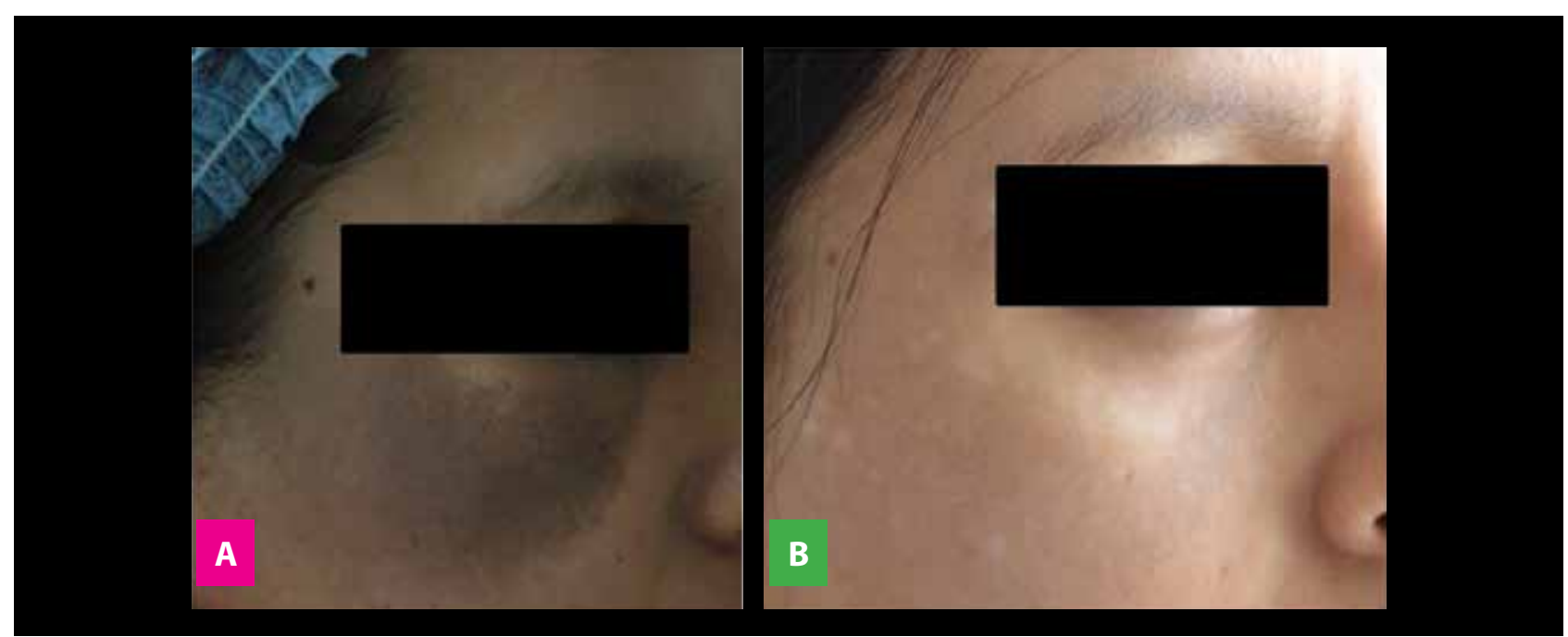

Figura 2. Paciente mujer de 22 años. La frecuencia de sus sesiones fue de 60 días y se realizaron 10 sesiones en total. Presentó efectos colaterales de hipopigmentación. No necesitó uso de despigmentante. A) Antes y B) Después del tratamiento. 


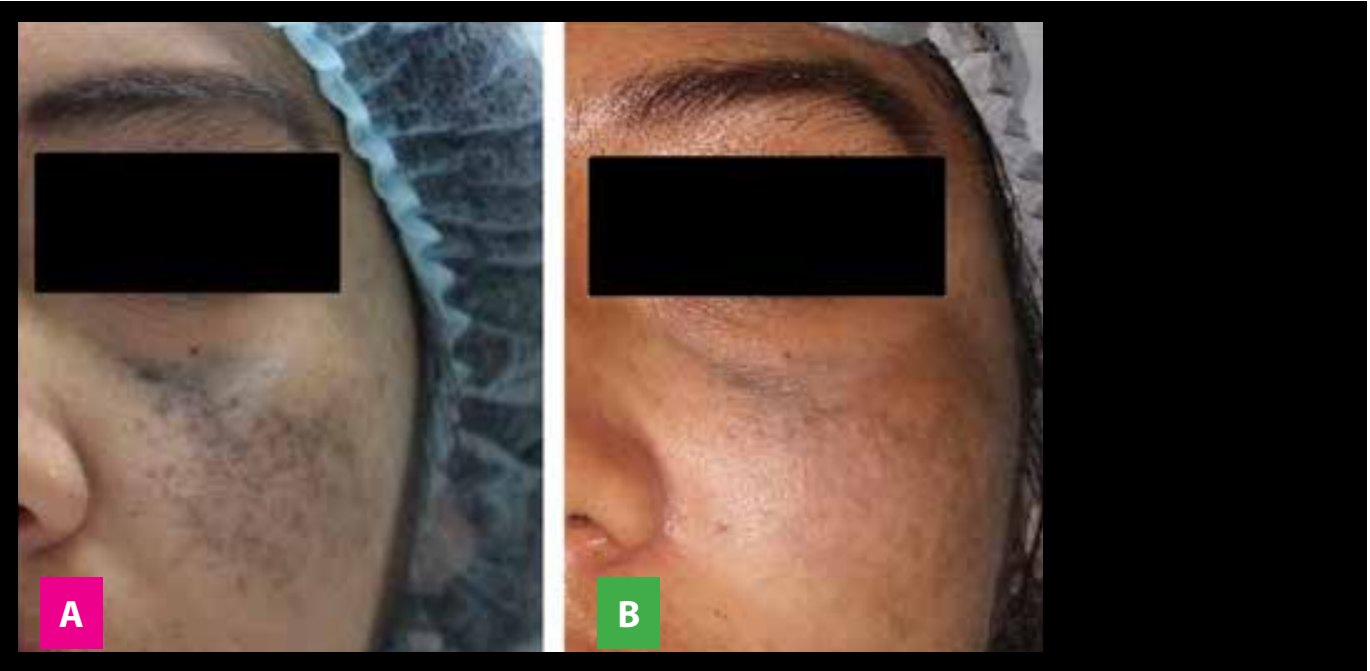

$\rightarrow$ Figura 3. Paciente mujer de 32 años. La frecuencia de sus sesiones fue de 60 días, se realizaron 8 sesiones en total. Presentó efectos colaterales de pigmentación post inflamatoria y necesitó despigmentante entre sesiones. A) Antes y B) Después del tratamiento.

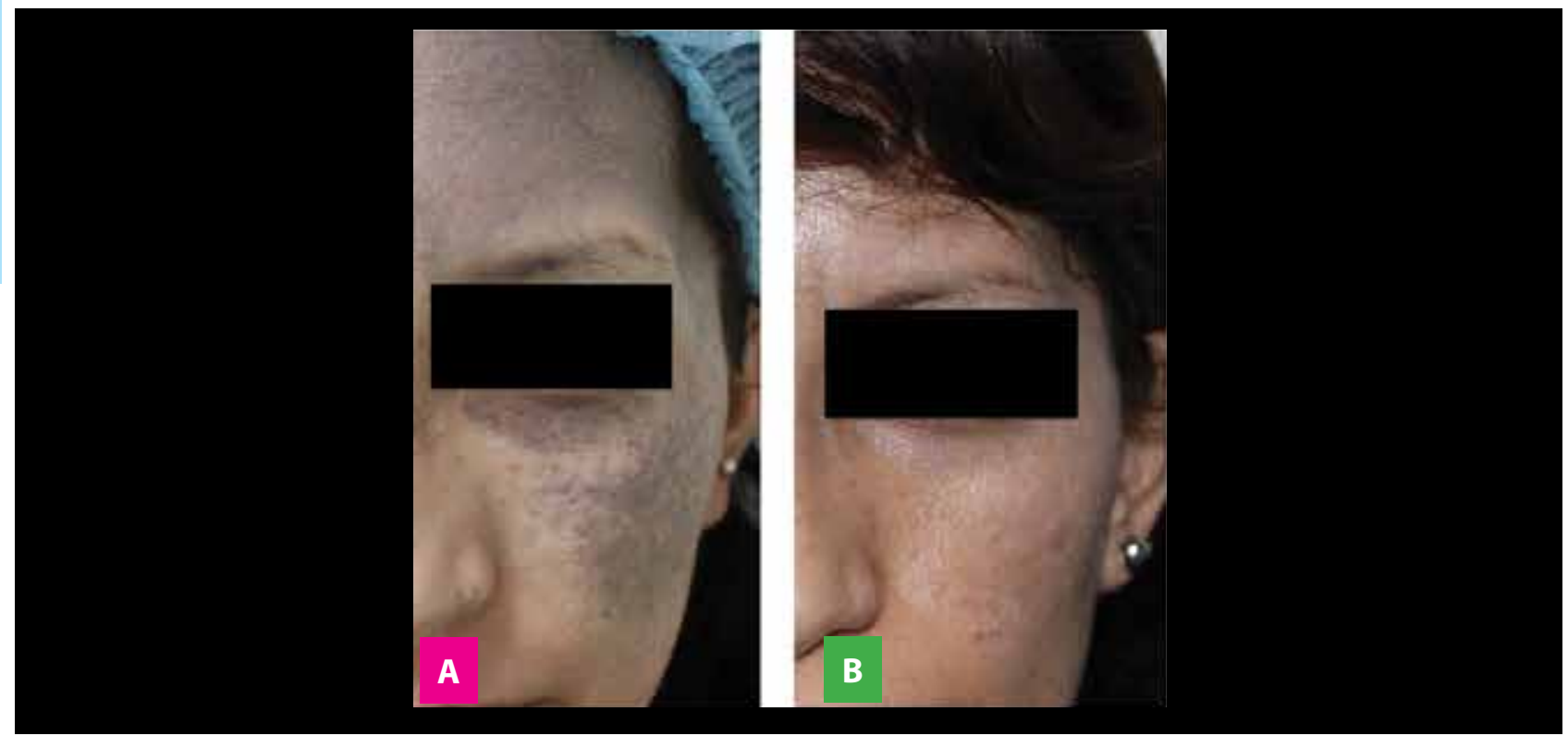

Figura 4. Paciente mujer de 29 años. La frecuencia de sus sesiones fue de 60 días, se realizaron 9 sesiones en total. No presentó efectos colaterales. A) Antes y B) Después del tratamiento.

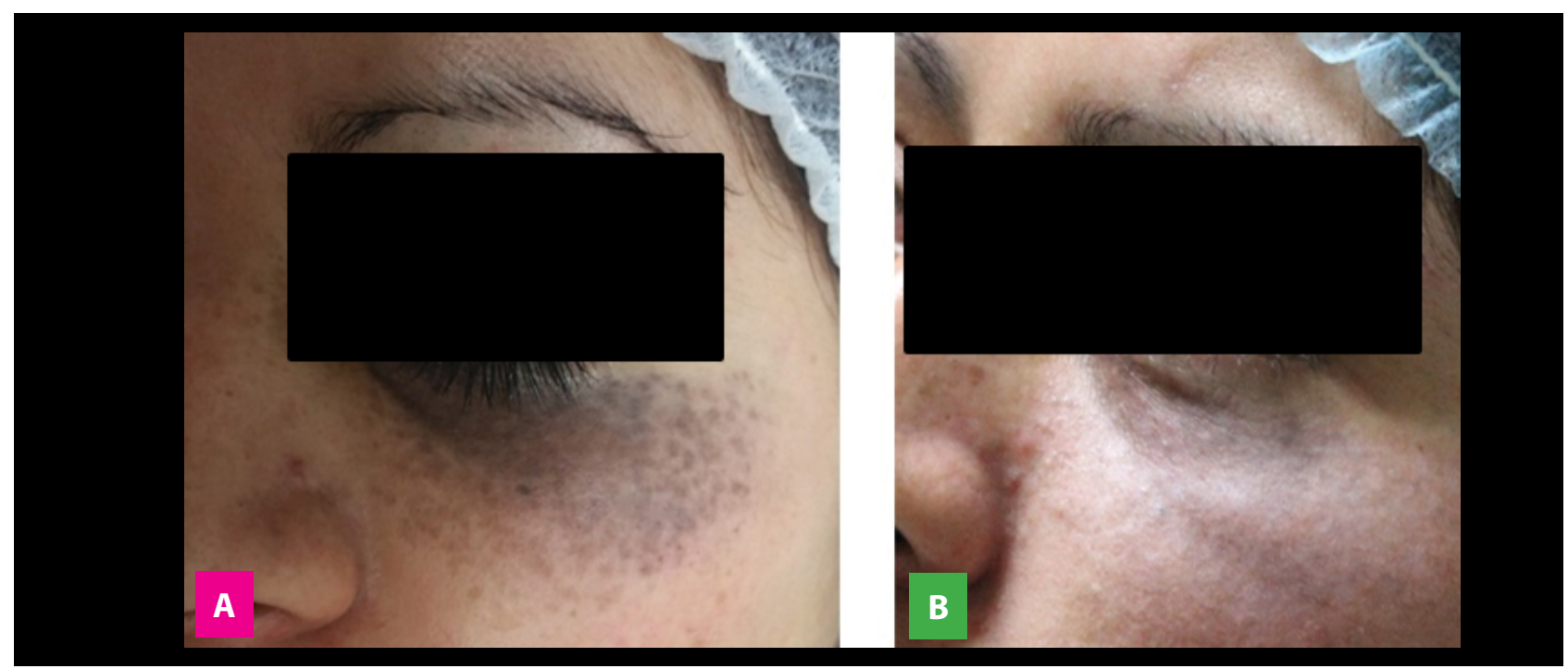

Figura 5. Paciente mujer de 18 años. La frecuencia de sus sesiones fue de 30 días y se realizaron 9 sesiones en total. No presentó efectos colaterales. A) Antes y B) Después del tratamiento. 


\section{DISCUSIÓN}

Existen múltiples tratamientos con la intensión de minimizar el impacto psicológico en los pacientes, pero además de ser poco eficaces, pueden formar cicatrices inestéticas que agravan aún más el problema. ${ }^{(11)}$ El láser ha revolucionado el tratamiento para estas lesiones, siendo el Q-switched el concepto de fototermólisis selectiva, el que genera mejores resultados a largo plazo. Esto ha hecho que se convierta en el tratamiento de elección para lesiones como el $\mathrm{NO}^{(16)}$.

Se hace evidente en este fototipo de piel que los efectos colaterales se presentan con facilidad, pero ha sido posible comprobar que las áreas de tratamiento despigmentadas no vuelven a pigmentarse; siempre y cuando el paciente tome las medidas de cuidado apropiadas, en caso contrario puede presentar lesiones hiperpigmentadas.

Los pacientes que completaron el tratamiento y siguieron las indicaciones no presentaron repigmentación en la lesión tratada, por lo cual el índice de satisfacción fue aceptable.

El número de sesiones es relativo en cada paciente, sin embargo, se ha evidenciado que el promedio para obtener resultados favorables varía entre 8 y 10 sesiones. El resultado a largo plazo (20 meses) es el mismo que se aplicó cada 30 días y cada 60 días.

Lo importante es que se pudo comprobar que la frecuencia de sesiones no alteró el resultado final de despigmentación y/o la aparición de efectos colaterales. En caso aparezcan lesiones colaterales recomendamos distanciar el periodo entre sesiones e iniciar protocolo de despigmentación (solo por las noches) con ácido kojico $2 \%$, ácido modélico $5 \%$, triamcinolona $0,025 \%$, la cual disminuye la posibilidad de aparición de lesiones permanentes hiperpigmentadas.
Sugerimos ampliar el universo de estudio en futuras investigaciones y contrastar los resultados, pudiendo colocar a esta tecnología lumínica como terapia de primera línea de este inesteticismo a veces incapacitante psicológicamente.

Los pacientes que han concluido el tratamiento mostraron alto grado de satisfacción por el aclaramiento de su lesión y una notable mejoría en su estado emocional. Esto fue comprobado durante una conversación con cada uno de los pacientes, quienes indicaron no tener ahora motivos para esconder su rostro en actividades sociales.

\section{CONCLUSIÓN}

El láser Q-switched Alexandrita de $755 \mathrm{~nm}$ aplicado con un mínimo 8 sesiones en un lapso no menor a 30 días con una intensidad de $6 \mathrm{~J} / \mathrm{cm} 2$ fue una terapia efectiva en casos de Nevus de Ota, logrando un alto grado de despigmentación. Por lo tanto, debería considerarse como tratamiento de primera línea para el manejo de esta patología.

Contribuciones de autoría: Los autores manifiestan no tener ningún interés comercial en los productos o aparatos reseñados en el presente trabajo.

Financiamiento: Autofinanciado.

Conflicto de interés: Los autores declaran no tener conflicto de interés en la publicación de este artículo.

Recibido: 08 agosto del 2019

Aprobado: 03 noviembre del 2019

Correspondencia: Julio Negrón.

Dirección: Calle Morisot 121 - San Borja, Lima - Perú.

Teléfono: +1923144951

Correo:julionegron@clinicalederma.pe

\section{REFERENCIAS BIBLIOGRÁFICAS}

1. Seo H-M, Choi C-W, Kim W-S. Beneticial effects of early treatment of nevus of Ota qith low fluence 1064nm q-switched Nd:Yag láser. Dermatol Surg. 2015; 41 (I): 142 148. doi: 10.1097/DSS.0000000000000212

2. Falabella R, Victoria, M. Barona, L. Fundamentos de Medicina: Dermatología, $7^{\circ}$ Edición 2009; 420-26. Disponible en: https://es.scribd.com/book/350142962 Dermatologia-Fundamentos-de-medicina-8\%C2\%AA-edicion

3. Alcala D, Valente I. Nevos melanociticos y no melanociticos. Revisión de la literatura. Rev Cent Dermatol Pascua. 2010; 19(2): 49-58. Disponlibe en: https:// www.medigraphic.com/pdfs/derma/cd-2010/cd102a.pdf

4. Hasson A, Navarrete-Dechent C, Nicklas C, Carreño N. Láser Q-Switched Nd:Yag $1064 \mathrm{~nm}$ para el tratamiento del Nevus de Ota: resultados en 22 pacientes y revisión de la literatura. Piel(Barc). 2012; 27(3): 113-17. Disponible en:https://dialnet.unirioja. es/servlet/articulo?codigo $=3955306$

5. Rex J, Ferrandiz C. Nevus Melanociticos. 185-91. Disponible en: https://www. aeped.es/sites/default/files/documentos/nevus.pdf

6. Shah V, Bray F, Aldahan A, Mlacker S, Nouri K. Lásers and Nevus of ota: a comprenhensive review. Lásers med Sci. 2016; 31(1): 179-85. doi: 10.1007/s10103015-1834-2

7. Saracoglu S. Case Report: Treatment of Nevus of Ota with 1064 nm Q-switched Nd:Yag. Journal of tha Láser and Health Academy. 2013; 2013(2):42-5. Disponible en: $\quad$ https://www.laserandhealthacademy.com/media/objave/academy/ priponke/42_45__case_report__saracoglu__qsw_ndyag_treatment_of nevus_of_ota.pdf

8. Hori Y, Kawashima M, Oohara K, Kukita A. Acquired, bilateral nevus of Ota- like macules. J Am Acad Dermatol. 1984 Jun;10(6):961-4. doi:10.1016/s01909622(84)80313-8

9. Stanford DG, Georgouras KE. Dermal melanocytosis: a clinical spectrum. Australas J Dermatol. 1996 Feb;37(1):19-25. Doi:10.1111/j.1440-0960.1996.tb00989.x

10. Henry H.L. Chan, MB, FRCPG, FRCPE, Taro Kono, MD. Nevus of Ota: Clinical Aspects and Management. SKINmed .2003; 2(2): 89-98. doi: 10.1111/j.15409740.2003.01706.

11. Toshio Kobayashi MD. Microsurgical Treatment of Nevus of Ota.

The Journal of Dermatologic Surgery and Oncology. 1991; 17(12): 936 - 41. doi: https://doi.org/10.1111/j.1524-4725.1991.tb01693.x

12. Watanabe S. Takahashi H. Treatment of nevus de Ota with Q-switched rubi laser. N Engl j Med. 1994; 331:1745-1750 doi: 10.1056/NEJM199412293312604

13. Chan $\mathrm{HH}$, Kono T. Nevus of Ota: clinical aspects and management. Skinmed. 2003 Mar-Apr; 2(2): 89-96; quiz 97-8. doi: 10.1111/j.1540-9740.2003.01706.x

14. Felton SJ, Al-Niaimi F, Ferguson JE, Madan V. our perspective of the treatment of naevus of ota with 1064 - 755 - and 532 nm wavelength láser. Láser Med Sci. 2013 29(5):1745-49 doi: $10.1007 / \mathrm{s} 10103-013-1332-3$

15. Choi J, Bong J, Beom K, Soon B, Yeo U, Hun C, Hoon J, Chul Y. A retrospective analysis of the clinical efficacies of q-switched alexandrite and q-switched $\mathrm{Nd}$-Yag lásers in the treatment of nevus of Ota in Korean patients. Journal of dermatologica treatment. 2015; 26(3): 240-45. doi: 10.3109/09546634.2014.930409

16. Aurangabadkar S. QYAG5 Q-switched Nd:Yag Láser Treatment of Nevus of Ota: An Indian Study of 50 Patients. J cutan Aesthet Surg. 2008; 1(2): 80-4.doi: 10.4103/0974-2077.44164 